\title{
RESEARCH
}

Open Access

\section{Pleiotropic association of LIPC variants with lipid and urinary 8-hydroxy deoxyguanosine levels in a Taiwanese population}

Ming-Sheng Teng ${ }^{1}$, Semon Wu ${ }^{1,2}$, Lung-An Hsu ${ }^{3}$, I-Shiang Tzeng ${ }^{1}$, Hsin-Hua Chou ${ }^{4}$, Cheng-Wen Su ${ }^{1}$ and Yu-Lin Ko $\mathrm{O}^{1,4,5^{*}}$

\begin{abstract}
Background: Hepatic lipase ( $\mathrm{HL}$, encoded by $L I P C$ ) is a glycoprotein primarily synthesized and secreted by hepatocytes. Previous studies had demonstrated that $\mathrm{HL}$ is crucial for reverse cholesterol transport and affects the metabolism, composition, and level of several lipoproteins. In current study, we investigated the association of LIPC (Lipase C, Hepatic Type) variants with circulating and urinary biomarker levels by using subgroup and mediation analyses.

Methods: A total of 572 participants from Taiwan were genotyped for three LIPC single nucleotide polymorphisms (SNPs) by using TaqMan assay. Fasting levels of glucose, lipid profile, inflammation markers, urine creatinine and 8hydroxy deoxyguanosine (8-OHdG) were measured. The chi-square test, 2-sample $t$ test and Analysis of variance (ANOVA) were used to examine differences among variables and genotype frequencies.

Results: SNPs rs2043085 and rs1532085 were significantly associated with urinary 8-OHdG levels, whereas all three SNPs were more significantly associated with Triglycerides (TG) or HDL-cholesterol (HDL-C) levels after additional adjustment for HDL-C or TG levels, respectively. Subgroup analyses revealed that the association of the LIPC SNPS with the levels of serum TG, HDL-C, and urinary 8-OHdG were predominantly observed in the men but not in the women. Differential associations of the LIPC SNPs with various lipid levels were observed in participants with different adiposity statuses. Mediation analyses indicated that TG levels acted as a suppressor masking the association of the LIPC genotypes with HDL-C levels, particularly in the men (Sobel test, all $P<0.01$ ).

Conclusion: Our data revealed that interaction and suppression effects mediated the pleiotropic association of the LIPC variants. The effects of the LIPC SNPs depended on sex, adiposity status, and TG levels. Thus, our findings can provide a method for identifying high-risk populations of cardiovascular diseases for clinical diagnosis.
\end{abstract}

Keywords: Hepatic lipase, Single-nucleotide polymorphism, Mediation analysis, Suppression effect, Triglyceride level, High-density lipoprotein cholesterol level

\footnotetext{
* Correspondence: yulinkotw@yahoo.com.tw

'Department of Research, Taipei Tzu Chi Hospital, Buddhist Tzu Chi Medical

Foundation, New Taipei city, Taiwan

${ }^{4}$ The Division of Cardiology, Department of Internal Medicine and

Cardiovascular Center, Taipei Tzu Chi Hospital, Buddhist Tzu Chi Medical

Foundation, New Taipei city, Taiwan

Full list of author information is available at the end of the article
}

(c) The Author(s). 2019 Open Access This article is distributed under the terms of the Creative Commons Attribution 4.0 International License (http://creativecommons.org/licenses/by/4.0/), which permits unrestricted use, distribution, and reproduction in any medium, provided you give appropriate credit to the original author(s) and the source, provide a link to the Creative Commons license, and indicate if changes were made. The Creative Commons Public Domain Dedication waiver (http://creativecommons.org/publicdomain/zero/1.0/) applies to the data made available in this article, unless otherwise stated. 


\section{Background}

Hepatic lipase (HL, encoded by LIPC) is a glycoprotein primarily synthesized and secreted by hepatocytes [1]. It is a member of the triacylglycerol lipase family responsible for the hydrolysis of TGs and phospholipids [2]. It converts large, TG-rich $\mathrm{HDL}_{2}$ into small, dense $\mathrm{HDL}_{3}$ and is a negative regulator of plasma HDL cholesterol (HDL-C) levels [1]. In addition, it facilitates the uptake of chylomicron remnant-like particles by acting as a ligand for glycosaminoglycans on the surface of rat hepatocytes [1]. In humans, its deficiency increases the levels of large $\mathrm{HDL}_{2}$ particles, enriches $\mathrm{HDL}$ with TG, and causes hyperalphalipoproteinemia [1]. Taken together, HL is crucial for reverse cholesterol transport and affects the metabolism, composition, and levels of several lipoproteins $[3,4]$.

Numerous genome-wide association studies (GWAS) have found the associations between LIPC SNPs and lipid levels, most commonly that of HDL-C, in different ethnic populations [5-7]. However, inconsistent results have been reported regarding the association of the LIPC promoter SNP C-514 T/rs1800588 with TG and HDL-C levels $[8,9]$. Interaction with sex, obesity, dietary intake, and physical activity further complicates these pleiotropic associations $[10,11]$. Several groups have attempted to increase the statistical power by including a high number of participants into meta-analyses or phenome-wide association studies (PheWASs). However, except for HDL-C, the association of rs1800588 with various lipids has still not been consistently replicated [12-14]. The source of complexity may originate from a plethora of confounding factors, and other SNPs in linkage disequilibrium with rs 1800588 may modify the association of rs1800588 with lipid traits.

HL activity has been linked to oxidative stress. For instance, the rs1800588-T allele is shown to reduce HL activity and increase the levels of malondialdehyde (MDA)-modified LDL [9], possibly via free radicals [15]. HL may affect the generation of reactive oxygen species (ROS) by modulating the activity of peroxisome proliferator-activated receptor $\delta$ (PPAR $\delta$ ), a key transcription factor known to counteract ROS production [16-18]. Because oxidized LDL are taken up by macrophage which enhanced foam cell formation, it is believed to increase the risk of systemic inflammation and atherosclerosis [19]. However, data from GWAS-related studies still have not provided concrete evidence for an association between rs1800588 and CAD [20, 21], and other LIPC SNPs facilitating the generation of oxidative stress signals may contribute to the risk of atherosclerosis and CAD.

Among all product of nucleic acids oxidation, 8-hydroxydeoxyguanosine ( $8-\mathrm{OHdG}$ ) is the most characterized. It is constantly excreted into the urine before and after a meal, and is stable in a freezer for up to a year [22]. High urinary 8-OHdG levels are positively associated with cancer, atherosclerosis, hypertension, chronic kidney diseases, and diabetes [23-27]. The ROS accumulates as these diseases progress, and the number of lesions escalates when more guanosine bases are damaged [28-30]. In contrast, urinary $8-\mathrm{OHdG}$ is negatively associated with BMI [31]. Nevertheless, the close interplay between oxidative stress and disease progression suggests the usefulness of 8-OHdG as a marker for both conditions.

In addition, HL activity may play a role in the generation of inflammation. Constant exposure of the endothelium to products of lipolysis through the action of various vascular lipases, including HL, could trigger pro- or anti-inflammatory responses in and around the endothelial cells [32], depending on the activation or inactivation of downstream signal pathways. Mice with HL deficiency had fewer number of macrophage in the adipose tissue and ameliorate inflammation and macrophage proliferation by inactivating the LIGHT/lymphotoxin $\beta$-receptor pathway, and lessen the burden of atherosclerosis in mice with metabolic syndrome or insulin resistance [33]. Andres-Blasco et al. have shown that HL(-/-) mice fed with a high-fat, high-cholesterol diet had phenotypes consistent with increased inflammation including enhanced circulating monocyte chemotactic protein 1 (MCP1) levels and activation of stress-induced SAPK/JNK- and p38-MAPK pathways [34]. Therefore, examining the correlation between the LIPC SNPs and inflammatory marker levels in patients with cardiovascular disease can help us understand the role and contribution of HL to the development of this disease.

To understand the role of LIPC SNPs in determining lipid profiles and to elucidate the mechanisms underlying their effects, we included three SNPs in the $5^{\prime}$ region (rs2043085, rs1800588, and rs1532085) and analyzed their associations and mediation effects with various lipid traits in a Taiwanese population. We determined whether these SNPs contributed to oxidative stress or inflammation by using urinary 8-OHdG as an oxidative stress marker [25] and analyzed the correlation between its levels and these LIPC SNPs. Because we had previously determined the interactive effect of sex and obesity on the association of two LIPC promoter SNPs with lipid traits [8], we performed subgroup and mediation analyses in this extended study of $L I P C$ variants.

\section{Methods}

\section{Subjects}

This study was approved by the Institutional Review Board of Taipei Tzu Chi Hospital, Buddhist Tzu Chi Medical Foundation. After obtaining informed consent, participants from the general population were consecutively recruited 
during routine cardiovascular health examinations. Only those without a known history of major systemic diseases or cardiovascular diseases were enrolled. Clinical history including hypertension, diabetes, habitual smoking, and drug use was recorded for all participants. Exclusion criteria included age $<18$ years, pregnancy, cancer, and a history of myocardial infarction, stroke, or transient ischemic attack. In addition, participants with diabetes mellitus (defined as blood sugar level before meals $\geq 7.0 \mathrm{mmol} / \mathrm{L}$ or regular use of medications for diabetes mellitus) and macroalbuminuria (defined as a urinary albumin-creatinine ratio $[\mathrm{ACR}]>300$ $\mathrm{mg} / \mathrm{g}$ ) were excluded. Furthermore, to prevent the enrollment of patients with acute inflammatory disease, those with CRP levels $>10 \mathrm{mg} / \mathrm{L}$ [35] and using lipid-lowering medications were excluded. In total, 572 participants (296 men with a mean age of $45.0 \pm 9.6$ years and 276 women with a mean age of $46.5 \pm 9.7$ years) were enrolled. Participants who smoked at least one cigarette per day at the time of the survey were considered current smokers.

\section{Genomic DNA extraction and genotyping}

Genomic DNA was extracted as previously described [36]. Three LIPC SNPs (rs2043085, rs1532085, and rs1800588) that have previously been found consistently associated with various metabolic phenotypes [7-11, 37] were selected according to the NCBI SNP database (http://www. ncbi.nlm.nih.gov/SNP). Genotyping was performed using TaqMan SNP Genotyping Assays from Applied Biosystems (ABI; Foster City, CA, USA). Basic characteristics and genotyping assays for the LIPC SNPs are listed in Additional file 1:Table S1. For quality control purposes, approximately $10 \%$ of the samples were re-genotyped blind, and identical results were obtained.

\section{Laboratory examinations}

A total of $15 \mathrm{~mL}$ of venous blood and urine were collected the morning after an overnight fast. Serum and plasma samples were obtained through centrifugation at $3000 \times \mathrm{g}$ for $15 \mathrm{~min}$ at $4{ }^{\circ} \mathrm{C}$. Immediately after centrifugation, the serum and plasma samples were frozen and stored at -80 ${ }^{\circ} \mathrm{C}$ prior to analyses. The circulating plasma levels of matrix metalloproteinase 2 (MMP2), soluble P-selectin (sP-selectin), and soluble TNF receptor II (sTNFRII) and the serum levels of matrix metalloproteinase 1 (MMP1) were measured using commercially available ELISA kits (R\&D, Minneapolis, MN, USA). Circulating serum levels of CRP were determined using the particle-enhanced turbidimetric immunoassay technique (Siemens Healthcare Diagnostics Ltd., Camberley, UK). The increase in turbidity that accompanies aggregation is proportional to the CRP concentration. Other markers including serum amyloid A (SAA), homocysteine, soluble intercellular adhesion molecule-1 (sICAM1), soluble vascular cell adhesion molecule-1 (sVCAM1), soluble E-selectin (sE-selectin), matrix metalloproteinase 9 (MMP9), plasma monocyte chemotactic protein 1 (MCP1), urine creatinine, and 8-OHdG were measured using a sandwich ELISA developed in-house. For the in-house ELISA, we coated the microwell of microtiter plates with primary anti-target protein antibody and detected the captured target protein with biotin-conjugated detecting antibody. To speed up the assay, we added the sample and biotin-conjugated detecting antibody simultaneously into the antibody-coated well. The final signal was provided by Amdext streptavidin HRP conjugate. All in-house kits exhibited satisfactory correlation when compared with commercially available ELISA kits (Additional file 1:references). Overall, the intraand inter-assay variability coefficients were within the range of 1.8 to $9.5 \%$. EDTA was used to prepare plasma for the analysis of sP-selectin, MMP-2 and sTNFRII, while sodium citrate was used to prepare plasma for the analysis of fibrinogen.

Glucose levels were enzymatically determined using the hexokinase method, and total cholesterol (TC) and TG levels were measured through automatic enzymatic colorimetry. HDL-C levels were enzymatically measured after phosphotungsten/magnesium precipitation. LDL-C levels were calculated using the Friedewald formula; however, in patients with a TG level $>400 \mathrm{mg} / \mathrm{dL}$, LDL-C levels were measured using commercial reagents with a standard protocol. Plasma fibrinogen levels were determined using the Clauss method adapted for a Sysmex CA1-1500 instrument (Kobe, Japan). Serum insulin levels were measured using an immunoradiometric assay (Bio-source, Nivelles, Belgium). The homeostasis model assessment of insulin resistance (HOMA-IR) index was calculated using the following formula: HOMA-IR = fasting serum insulin $(\mu \mathrm{U} / \mathrm{mL}) \times$ fasting plasma glucose $(\mathrm{mmol} / \mathrm{L}) / 22.5$.

\section{Statistical analysis}

The chi-square test was used to examine differences among categorical variables and to compare allele and genotype frequencies. The clinical characteristics of continuous variables were expressed as means \pm standard deviations and tested using the 2-sample $t$ test or ANOVA. A general linear model was applied to identify the major effect of each polymorphism on clinical phenotype variables, with age, sex, BMI, and smoking as confounding covariates. All biomarker levels were logarithmically transformed prior to statistical analyses to adhere to a normality assumption. The biomarkers had non-normal distributions after normality test were analyzed by using Kolmogorov-Smirnov test. The result was adjusted by false discovery rate (FDR) for multiple test correction and the regression coefficient with FDR $P$ value $<0.05$ was considered as significant. The analysis of deviation from the Hardy-Weinberg equilibrium 
(HWE) and the estimation of linkage disequilibrium between polymorphisms were performed using Golden Helix SVS Win32 7.3.1 (Golden Helix, Bozeman, MT). For multiple testing in the genetic association analysis, values of $P \leq 0.005$ using a 2 -sided test were considered statistically significant. Missing data were handled with listwise deletion. To investigate mediation effects exerted by TG levels on the association of the LIPC variants with HDL-C levels, a conceptual model was proposed, and four criteria were devised to evaluate the suppression effects of serum TG levels [38]. Criterion one: The independent variable (LIPC genotypes) must predict the mediator (TG levels). Criterion two: The mediator (TG levels) must predict the dependent variable (HDL-C) when adjusting for the independent variable. The mediation effect was calculated as the product of two regression coefficients from criteria one and two and reflected intermediate pathways from the independent variable to the dependent variable through the mediator. The regression coefficient denoting a relationship between the independent and dependent variables adjusted for the mediator was expressed as a direct effect. Criterion three: The independent variable must have a significant effect on the dependent variable and be expressed as the total effect. The total effect could also be obtained by summing the direct and mediation (indirect) effects. Criterion four: The mediation effect must be significant according to the procedure outlined by Sobel $[39,40]$. In addition, a suppression effect may arise in a situation when the direct effect was greater than the total effect [41]. In this situation, the direct and indirect effects often had fairly similar magnitudes with opposite signs, which were entirely or partially offset, resulting in a zero or a nonzero but ultimately insignificant total effect [42]. The Sobel test [43] is the most commonly used method for examining the statistical significance of the mediation effect. Therefore, we used $\beta$ coefficients and standard errors from the above model to perform the Sobel test. The Sobel test was performed using an interactive tool (http://www.quantpsy.org/sobel/sobel.htm) by which the null hypothesis $\left(\mathrm{H}_{0}: \alpha \beta=0\right)$ was tested. The test statistic $\mathrm{S}$ approximately distributed as $\mathrm{Z}$ was obtained by dividing the estimated mediation effect $(\alpha \beta)$ with the standard error $(\delta)$. The reported $P$ values were drawn from the unit normal distribution under the assumption of a $\mathrm{Z}$ value in which the mediated effect equaled zero in the population, with \pm 1.96 critical values containing the central $95 \%$ of the unit normal distribution.

\section{Results}

\section{Baseline data}

Demographic data, clinical biochemical data, lipid and inflammatory biomarker profiles, and urinary 8-OHdG levels of the participants, stratified by sex, are summarized in Table 1. A significantly higher percentage of the men were current smokers $(P<0.001)$. In addition, the men had significantly higher BMI $(P<0.001)$, HOMA-IR $(P<0.001)$, waist circumference $(P<0.001)$, waist/hip ratio $(P<0.001)$, and circulating levels of fasting plasma glucose $(P<0.001)$, serum insulin $(P=0.01)$, LDL-C $(P=$ 0.036), TG $(P<0.001)$, sE-selectin $(P<0.001)$, sP-selectin $(P=0.005)$, MMP-9 $(P=0.003)$, sTNFRII $(P=0.018)$, and MCP-1 $(<0.001)$ than did the women. By contrast, circulating HDL-C $(P<0.001)$, MMP-2 levels $(P=0.018)$ and urine 8-OHdG levels $(P<0.001)$ were lower in the men than in the women. For the studied polymorphisms, no significant difference in genotype frequencies between the men and women and no significant deviation from the HWE were observed (Additional file 1: Table S1).

\section{Relationship between the LIPC SNPs and lipid variables}

To determine the effects of the LIPC genotypes on lipid levels, we created an additive model by using four lipid traits as variables of interest (Table 2). After adjustment for age, sex, smoking status, and BMI, compared with the participants carrying the major alleles of the studied SNPs, those carrying the minor alleles exhibited a trend of higher TG levels $(P=0.007, P=0.006$, and $P=0.009$ for rs2043085, rs1532085, and rs1800588, respectively). The association became more significant after further adjustment for HDL-C levels and FDR corrections $(P=$ $2.7 \times 10^{-4}, P=2.7 \times 10^{-4}$, and $P=4.5 \times 10^{-4}$, respectively). Although unadjusted $P$ values were bordering the cutoff threshold, the association of the studied SNPs with HDL-C levels became much more significant after further adjustment for serum TG levels and FDR corrections $\left(P=5.4 \times 10^{-4}, P=4.5 \times 10^{-4}\right.$, and $P=0.002$ for rs2043085, rs1532085, and rs1800588, respectively). In addition, a significant association of the rs 1800588 genotype with TC levels was observed $(P=0.003)$.

\section{Associations of the LIPC SNPs with inflammatory biomarkers and urinary $8-\mathrm{OHdG}$ levels}

To determine whether the LIPC genotypes influenced inflammatory marker levels, we analyzed the levels of the following 12 inflammatory markers: CRP, fibrinogen, SAA, sICAM1, sVCAM1, sE-selectin, sP-selectin, MMP1, MMP-2, MMP-9, MCP1, and sTNFRII. No significant differences were observed in all the analyzed inflammatory marker levels among the LIPC genotypes (Additional file 1: Table S2-S4). Table 2 lists the variations in urinary 8-OHdG levels across the LIPC genotypes. After the additive model was adjusted for age, sex, smoking status, and BMI, rs2043085 and rs1532085 genotypes were significantly associated with urinary 8-OHdG levels $(P=0.001$ and $P=0.002$, respectively). By 
Table 1 Clinical and biochemical characteristics of the study population

\begin{tabular}{|c|c|c|c|c|}
\hline & Total & Men & Women & $P$ value \\
\hline Number & 572 & 296 & 276 & \\
\hline Age (years) & $45.7 \pm 9.7$ & $45.0 \pm 9.6$ & $46.5 \pm 9.7$ & 0.076 \\
\hline BMI $\left(\mathrm{kg} / \mathrm{m}^{2}\right)$ & $24.2 \pm 3.4$ & $24.9 \pm 3.0$ & $23.5 \pm 3.6$ & $<0.001$ \\
\hline Fasting plasma glucose (mg/dL) & $92.0(88.0,98.0)$ & $94.0(89.0,100.0)$ & $90.5(86.0,96.0)$ & $<0.001$ \\
\hline Fasting serum insulin ( $\mu \mathrm{U} / \mathrm{mL})$ & $7.8(6.1,10.6)$ & $8.3(6.4,11.4)$ & $7.5(5.9,10.3)$ & 0.01 \\
\hline HOMAIR & $1.8(1.4,2.5)$ & $2.0(1.5,2.6)$ & $1.7(1.3,2.4)$ & $<0.001$ \\
\hline Waist circumference (cm) & $84.8 \pm 9.4$ & $87.7 \pm 7.4$ & $81.7 \pm 10.3$ & $<0.001$ \\
\hline Waist hip ratio & $0.9 \pm 0.1$ & $0.9 \pm 0.0$ & $0.8 \pm 0.1$ & $<0.001$ \\
\hline Current smokers (\%) & 19.4 & 33.8 & 4.0 & $<0.001$ \\
\hline Hypertension (\%) & 8.9 & 8.0 & 9.8 & 0.309 \\
\hline Diabetes mellitus (\%) & 3.3 & 4.1 & 2.5 & 0.312 \\
\hline Total cholesterol (mg/dL) & $199.3 \pm 35.9$ & $201.6 \pm 35.8$ & $196.8 \pm 36.0$ & 0.089 \\
\hline LDL-C (mg/dL) & $116.5 \pm 32.9$ & $119.4 \pm 33.7$ & $113.5 \pm 31.8$ & 0.036 \\
\hline HDL-C (mg/dL) & $54.0(45.0,65.0)$ & $48.0(42.0,55.0)$ & $61.0(51.3,71.0)$ & $<0.001$ \\
\hline $\mathrm{TG}(\mathrm{mg} / \mathrm{dL})$ & $114.5(76.0,164.8)$ & $135.0(92.0,203.8)$ & $91.0(67.0,134.8)$ & $<0.001$ \\
\hline CRP (mg/L) & $0.6(0.3,1.2)$ & $0.6(0.3,1.2)$ & $0.5(0.2,1.2)$ & 0.098 \\
\hline Fibrinogen (mg/dL) & $260.3 \pm 67.3$ & $256.9 \pm 68.8$ & $263.8 \pm 65.6$ & 0.187 \\
\hline sE-selectin(ng/L) & $49.9(35.6,64.3)$ & $57.0(41.6,73.5)$ & $42.5(31.1,54.7)$ & $<0.001$ \\
\hline sP-selectin(ng/mL) & $95.0(65.9,169.7)$ & $103.9(70.0,187.8)$ & $86.7(60.0,151.3)$ & 0.005 \\
\hline SAA $(\mu \mathrm{mol} / \mathrm{L})$ & $3.6(1.7,6.0)$ & $3.3(1.6,6.0)$ & $3.6(1.7,6.1)$ & 0.399 \\
\hline sICAM1 (ng/L) & $228.0(179.9,275.1)$ & $232.0(182.0,283.6)$ & $225.1(175.8,271.6)$ & 0.776 \\
\hline sVCAM1(ng/L) & $477.0(406.0,545.0)$ & $479.0(399.0,555.0)$ & $476.0(415.0,540.0)$ & 0.81 \\
\hline MMP-1 (pg/mL) & $188.3(102.1,398.8)$ & $184.8(110.8,404.4)$ & $189.0(96.2,398.4)$ & 0.601 \\
\hline MMP-2 (ng/mL) & $122.2(103.1,140.4)$ & $118.9(98.7,137.5)$ & $124.9(106.9,146.1)$ & 0.018 \\
\hline MMP-9 (mg/L) & $109.9(73.8,167.3)$ & $120.5(83.3,184.6)$ & $99.4(66.1,150.2)$ & 0.003 \\
\hline MCP-1 (pg/mL) & $59.8(43.6,83.5)$ & $62.6(46.1,86.7)$ & $56.2(40.9,80.8)$ & 0.005 \\
\hline sTNFRII (pg/mL) & $3095.8(2635.9,3708.9)$ & $3120.2(2742.4,3726.3)$ & $3066.8(2528.2,3700.6)$ & 0.018 \\
\hline 8-OHdG/creatinine (ng/mg) & $32.6(24.3,44.2)$ & $30.4(22.5,40.0)$ & $35.2(26.8,48.4)$ & $<0.001$ \\
\hline \multicolumn{5}{|l|}{ LIPC-rs2043085 } \\
\hline CC & $159(28.3 \%)$ & $73(25.2 \%)$ & $86(31.6 \%)$ & \multirow[t]{3}{*}{0.198} \\
\hline TC & $284(50.5 \%)$ & $150(51.7 \%)$ & $134(49.3 \%)$ & \\
\hline$\pi$ & $119(21.2 \%)$ & $67(23.1 \%)$ & 52 (19.1\%) & \\
\hline \multicolumn{5}{|l|}{ LIPC-rs1532085 } \\
\hline GG & $162(28.8 \%)$ & 74 (25.5\%) & 88 (32.2\%) & \multirow[t]{3}{*}{0.200} \\
\hline GA & $287(51.0 \%)$ & $153(52.8 \%)$ & 134 (49.1\%) & \\
\hline $\mathrm{AA}$ & $114(20.2 \%)$ & $63(21.7 \%)$ & 51 (18.7\%) & \\
\hline \multicolumn{5}{|l|}{ LIPC-rs1800588 } \\
\hline CC & $228(40.5 \%)$ & $125(43.1 \%)$ & $103(37.7 \%)$ & \multirow[t]{3}{*}{0.659} \\
\hline TC & $261(46.4 \%)$ & $128(44.1 \%)$ & $133(48.7 \%)$ & \\
\hline$\pi$ & 74 (13.1\%) & $37(12.8 \%)$ & 37 (13.6\%) & \\
\hline
\end{tabular}

BMI body mass index, HOMA-IR homeostasis model assessment of insulin resistance, HDL-C high-density lipoprotein cholesterol, LDL-C low-density lipoprotein cholesterol, TG Triglycerides, CRP C-reactive protein, sE-selectin soluble E-selectin, sP-selectin soluble P-selectin, SAA serum amyloid A, s/CAM1 soluble intercellular adhesive molecule 1, sVCAM1 soluble vascular cell adhesive molecule 1, MMP1 matrix metalloproteinase 1, MMP2 matrix metalloproteinase 2, MMP9 matrix metalloproteinase 9, MCP-1 Monocyte chemotactic protein-1, sTNFRIl soluble tumor necrosis factor-alpha receptor 2, 8OHdG 8-hydroxy deoxyguanosine. Continuous variables are presented as mean \pm SD. Fasting plasma glucose, fasting serum insulin, HOMAIR, HDL-C, TG, CRP, sE-selectin, sP-selectin, SAA, sICAM1, sVCAM1, MMP1, MMP2, MMP9, MCP1, sTNFRII and 8-OHdG values were logarithmically transformed before statistical testing to meet the assumption of normal distributions; however, the untransformed data are shown as median (range)

All variables had non-normal distributions after Normality test, including fasting plasma glucose, fasting serum insulin, HOMAIR, sE-selectin, sP-selectin, SAA, sICAM1, MMP1, MMP2, MMP9, MCP1 and sTNFRII were analyzed by using Kolmogorov-Smirnov test 
Table 2 Associations between the LIPC SNPs and lipid profiles and urinary $80 \mathrm{HdG}$ levels

\begin{tabular}{|c|c|c|c|c|c|}
\hline Genotypes & $\mathrm{MM}$ & $\mathrm{Mm}$ & $\mathrm{mm}$ & $\begin{array}{l}P 1 \\
\text { (adjusted } P \text { ) }\end{array}$ & $\begin{array}{l}P 2 \\
\text { (adjusted P) }\end{array}$ \\
\hline rs2043085, number & 159 & 284 & 119 & & \\
\hline Total cholesterol (mg/dL) & $198.8 \pm 39.7$ & $198.3 \pm 33.6$ & $201.6 \pm 35.8$ & 0.435 & \\
\hline $\mathrm{LDL}-\mathrm{C}(\mathrm{mg} / \mathrm{dL})$ & $120.2 \pm 37.9$ & $114.8 \pm 30.2$ & $115.2 \pm 31.4$ & 0.248 & \\
\hline $\mathrm{HDL}-\mathrm{C}(\mathrm{mg} / \mathrm{dL})$ & $53.0(44.0,64.0)$ & $53.0(44.0,66.0)$ & $54.0(45.0,65.0)$ & 0.039 & $\begin{array}{l}a_{3 \times 10^{-4}} \\
\left(5.4 \times 10^{-4}\right)\end{array}$ \\
\hline $\mathrm{TG}(\mathrm{mg} / \mathrm{dL})$ & $102.0(71.0,151.0)$ & $111.0(75.3,161.8)$ & $129.0(90.0,186.0)$ & $\begin{array}{l}0.007 \\
(0.021)\end{array}$ & $\begin{array}{l}{ }^{b_{6}} 6 \times 10^{-5} \\
\left(2.7 \times 10^{-4}\right)\end{array}$ \\
\hline 8-OHdG (ng/mg) & $32.9(21.8,44.1)$ & $31.6(24.7,43.0)$ & $35.9(27.4,47.6)$ & $\begin{array}{l}0.001 \\
(0.015)\end{array}$ & $\begin{array}{l}a_{4} \times 10^{-4} \\
\left(6.0 \times 10^{-4}\right)\end{array}$ \\
\hline rs1532085, number & 162 & 287 & 114 & & \\
\hline Total cholesterol (mg/dL) & $198.1 \pm 40.0$ & $198.6 \pm 33.3$ & $201.7 \pm 35.9$ & 0.309 & \\
\hline $\mathrm{LDL}-\mathrm{C}(\mathrm{mg} / \mathrm{dL})$ & $119.5 \pm 38.2$ & $115.2 \pm 30.0$ & $114.9 \pm 31.3$ & 0.348 & \\
\hline $\mathrm{HDL}-\mathrm{C}(\mathrm{mg} / \mathrm{dL})$ & $53.0(44.0,63.3)$ & $53.0(44.0,66.0)$ & $54.0(45.0,65.3)$ & 0.035 & $\begin{array}{l}a_{2} \times 10^{-4} \\
\left(4.5 \times 10^{-4}\right)\end{array}$ \\
\hline $\mathrm{TG}(\mathrm{mg} / \mathrm{dL})$ & $101.5(70.8,151.5)$ & $112.0(76.0,161.0)$ & $129.0(90.0,196.3)$ & $\begin{array}{l}0.006 \\
(0.021)\end{array}$ & $\begin{array}{l}\mathrm{b}_{5} \times 10^{-5} \\
\left(2.7 \times 10^{-4}\right)\end{array}$ \\
\hline 8-OHdG (ng/mg) & $32.6(22.4,44.1)$ & $32.1(24.8,43.5)$ & $35.8(26.9,46.6)$ & $\begin{array}{l}0.002 \\
(0.015)\end{array}$ & $\begin{array}{l}{ }^{a} 0.001 \\
(0.001)\end{array}$ \\
\hline rs1800588, number & 228 & 261 & 74 & & \\
\hline Total cholesterol (mg/dL) & $195.2 \pm 35.3$ & $200.3 \pm 36.1$ & $206.7 \pm 35.7$ & $\begin{array}{l}0.003 \\
(0.015)\end{array}$ & \\
\hline LDL-C (mg/dL) & $115.2 \pm 33.6$ & $116.6 \pm 32.7$ & $118.9 \pm 31.1$ & 0.196 & \\
\hline $\mathrm{HDL}-\mathrm{C}(\mathrm{mg} / \mathrm{dL})$ & $52.0(44.3,64.0)$ & $54.0(44.0,65.0)$ & $56.5(46.8,69.0)$ & 0.089 & $\begin{array}{l}{ }^{a} 0.002 \\
(0.002)\end{array}$ \\
\hline $\mathrm{TG}(\mathrm{mg} / \mathrm{dL})$ & $107.0(72.3,161.8)$ & $116.0(76.0,164.5)$ & $118.5(79.0,183.5)$ & $\begin{array}{l}0.009 \\
(0.023)\end{array}$ & $\begin{array}{l}{ }^{{ }} 2 \times 10^{-4} \\
\left(4.5 \times 10^{-4}\right)\end{array}$ \\
\hline 8-OHdG (ng/mg) & $32.4(24.3,43.3)$ & $33.7(25.5,45.1)$ & $29.7(22.3,39.4)$ & 0.155 & ${ }^{a} 0.177$ \\
\hline
\end{tabular}

Abbreviations as in Table 1

$P$ 1: adjusted for age, sex, BMI, and current smoke; $P 2$ : $P$ values of associations between SNPs and HDL-C, 8-OHdG or TG levels after adjustment for age, sex, BMI, current smoke and TG $\left({ }^{a}\right)$ or HDL-C $\left({ }^{b}\right)$ levels

MM: homozygosity of major allele, Mm: heterozygosity, mm: homozygosity of minor allele

Adjusted $P$ values were shown with False Discovery Rate correction of $P 1$ and $P 2$ values, and only significant $P$ values were demonstrated

contrast, no significant association of the rs1800588 genotype with urinary $8-\mathrm{OHdG}$ levels was observed.

\section{Interaction of the LIPC SNPs, lipid variables, and urinary 8-OHdG levels with sex}

As depicted in Table 3, in the additive model, the men carrying the minor alleles of the studied SNPs had significantly higher TG levels than did those carrying the major alleles $(P=0.005, P=0.005$, and $P=0.004$ for rs2043085, rs1532085, and rs1800588, respectively), and the associations became more significant after further adjustment for HDL-C levels and FDR corrections $(P=$ $6 \times 10^{-4}, P=4.5 \times 10^{-4}$, and $P=3.6 \times 10^{-4}$, respectively). By contrast, HDL-C levels were significantly associated with the LIPC genotypes in the men only after adjustment for serum TG levels and FDR corrections $(P=$ $0.006, P=0.005$, and $P=0.002$ for rs 2043085 , rs 1532085 , and rs1800588, respectively). In addition, after adjusting age, sex, smoking status, and BMI for the men, the rs2043085 genotype was significantly associated with urinary 8-OHdG levels $(P=0.001)$, whereas the rs1532085 genotype demonstrated a trend of the association $(P=0.007)$. Furthermore, a significant association of the rs 1800588 genotype with TC levels was observed only in the men $(P=0.003)$. None of the above associations was observed in the women after FDR correction. These results suggest that the associations of the LIPC SNPs with the lipid variables and urinary 8-OHdG levels were dependent on sex.

Interaction of the LIPC SNPs, lipid variables, and urinary 8-OHdG levels with obesity

We stratified the participants according to their adiposity statuses. As illustrated in Table 4, in the additive model, only the nonobese carriers of the minor alleles of rs2043085 and rs1532085 had significantly higher TG levels than did those carrying the major alleles $(P=0.002$ and $P=0.002$, respectively). By contrast, only the obese 


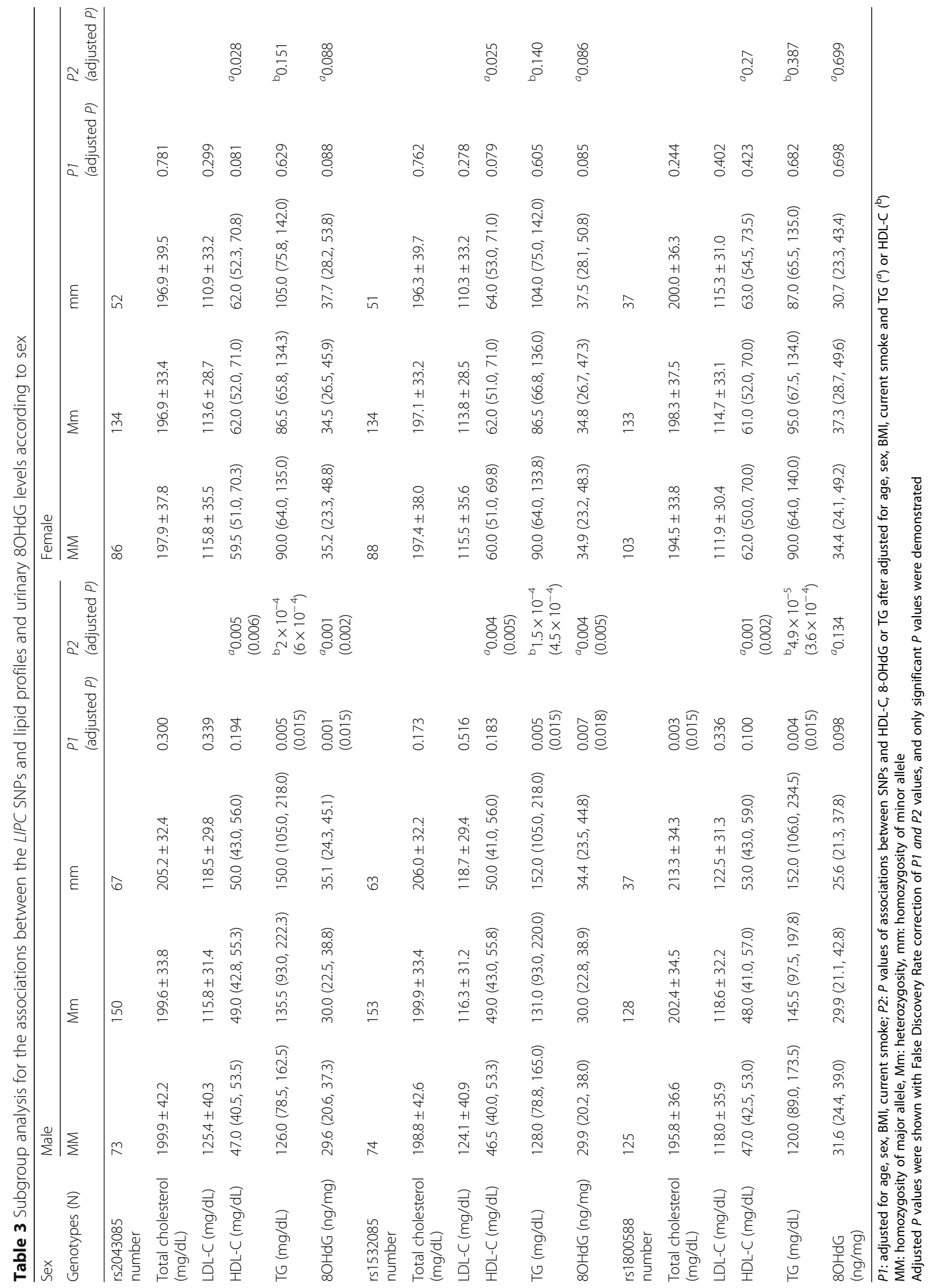




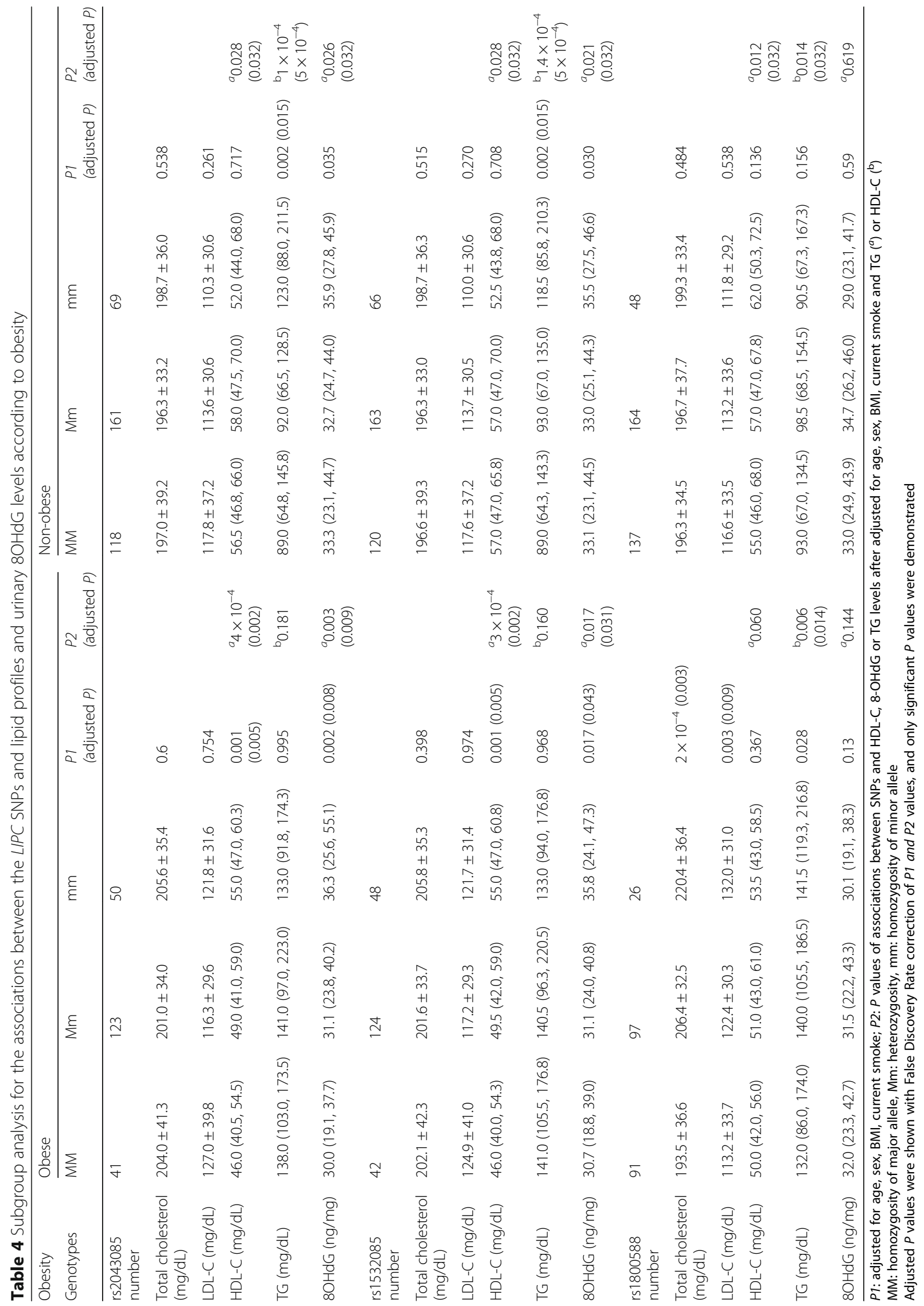


Table 5 Mediation tests of the TG levels on the associations between the LIPC genotypes and HDL-C levels in male participants

\begin{tabular}{|c|c|c|c|c|}
\hline & & rs2043085 & rs1800588 & rs1532085 \\
\hline Criterion 1 & $\begin{array}{l}\text { a } \\
\text { regression coefficient } \\
\text { Standard error } \\
P \text { value }\end{array}$ & $\begin{array}{l}0.059 \\
0.021 \\
0.005\end{array}$ & $\begin{array}{l}0.063 \\
0.021 \\
0.004\end{array}$ & $\begin{array}{l}0.061 \\
0.021 \\
0.005\end{array}$ \\
\hline Criterion 2 & $\begin{array}{l}\beta \\
\text { regression coefficient } \\
\text { Standard error } \\
{ }^{\#} P \text { value }\end{array}$ & $\begin{array}{l}-0.181 \\
0.021 \\
7.2 \times 10^{-16}\end{array}$ & $\begin{array}{l}-0.183 \\
0.021 \\
2.9 \times 10^{-16}\end{array}$ & $\begin{array}{l}-0.181 \\
0.021 \\
6.5 \times 10^{-16}\end{array}$ \\
\hline & $\begin{array}{l}\gamma^{\prime} \\
\text { regression coefficient } \\
\text { Standard error } \\
{ }^{*} P \text { value }\end{array}$ & $\begin{array}{l}0.022 \\
0.008 \\
0.005\end{array}$ & $\begin{array}{l}0.026 \\
0.008 \\
0.001\end{array}$ & $\begin{array}{l}0.022 \\
0.008 \\
0.004\end{array}$ \\
\hline Criterion 3 & $\begin{array}{l}a \beta+\gamma^{\prime} \\
\text { regression coefficient } \\
\text { Standard error } \\
P \text { value }\end{array}$ & $\begin{array}{l}0.011 \\
0.008 \\
0.194\end{array}$ & $\begin{array}{l}0.014 \\
0.009 \\
0.100\end{array}$ & $\begin{array}{l}0.011 \\
0.009 \\
0.183\end{array}$ \\
\hline Criterion 4 & $\begin{array}{l}a \beta \\
\text { regression coefficient } \\
\text { Standard error } \\
P \text { value (Sobel test) }\end{array}$ & $\begin{array}{l}-0.011 \\
0.004 \\
0.008\end{array}$ & $\begin{array}{l}-0.012 \\
0.004 \\
0.005\end{array}$ & $\begin{array}{l}-0.011 \\
0.004 \\
0.006\end{array}$ \\
\hline
\end{tabular}

LIPC genotypes were analyzed in dominant models,

a: unstandardized coefficient for the association between LIPC genotypes and TG levels

$\beta$ : unstandardized coefficient for the association between TG and HDL-C levels (when adjusting for LIPC genotypes)

Direct effect $=\gamma^{\prime}$, Total effect $=\alpha \beta+\gamma^{\prime}$, Mediation (indirect) effect $=\alpha \beta$ $P$ : adjusted for age, sex, BMI, current smoke; ${ }^{*}$ : indicated the $P$ value of association between SNPs and HDL-C after adjusted for age, sex, BMI, current smoke and TG; ${ }^{\#}$ : indicated the $P$ value of association between TG and HDL-C after adjusted for age, sex, BMI, current smoke and SNPs

carriers of the minor alleles of rs 2043085 and rs1532085 had at least a trend of significantly higher HDL-C and urinary 8-OHdG levels than did those carrying the major alleles $(P=0.001$ and $P=0.001$ for HDL-C levels and $P=0.002$ and $P=0.017$ for urinary 8-OHdG levels, respectively). Furthermore, a significant association of the rs 1800588 genotype with TC and LDL-C levels was observed only in the obese participants $\left(P=2 \times 10^{-4}\right.$ and $P=0.003$, respectively). These results suggest that differential associations of the LIPC SNPs with the lipid variables and urinary 8-OHdG levels were dependent on adiposity status.

\section{Serum TG levels suppressed the sex-dependent association of the LIPC SNPs with HDL-C levels}

Four criteria were applied to study the mediation and suppression effects in the men carrying different LIPC genotypes (Table 5). In brief, the LIPC genotypes were significantly associated with TG levels (criterion 1), which in turn had significant negatively effects on HDL-C levels (criterion 2). The total effect of the LIPC genotypes on HDL-C levels was $0.011,0.014$, and 0.011 for rs2043085, rs1800588, and rs1532085, respectively, with insignificant $P$ values (criterion 3 ). The Sobel test for the mediation of the results of the corresponding HDL-C levels revealed that $\mathrm{z}$ was $-2.8,-3$, and -2.8 , respectively (all $P<0.01$, criterion 4 ). Moreover, the direct effects $\left(\gamma^{\prime}\right)$ of the LIPC genotypes on HDL-C levels were higher than their total effects $\left(\alpha \beta+\gamma^{\prime}\right)$, which had similar magnitudes as those of the mediation effects but with opposite signs $(\alpha \beta)$, demonstrating a suppression effect in this model (Fig. 1).

\section{Discussion}

This study investigated the association of LIPC SNPs with various circulating lipid and biomarker levels. Our data revealed a significant association of three LIPC SNPs with TC, HDL-C, and TG levels, with the interaction of sex and obesity; this finding was consistent with that of our previous study [8]. In addition, we observed that TG levels exerted a suppression effect on the association of LIPC SNPs with HDL-C levels. Furthermore, the association of two LIPC SNPs with urinary 8-OHdG levels, a marker of systemic oxidative stress, was observed. The involvement of both pro- and anti-atherosclerotic risk factors observed in this study may have meaningful implications for preventive medicine. Our findings might facilitate the identification of high-risk populations in preventive medicine of cardiovascular diseases in the future.

To the best of our knowledge, this is the first study to report an association of two LIPC SNPs, rs2043085 and rs1532085, with urinary 8-OHdG levels. The SNP rs2043085 has been associated with both HDL-C and fasting plasma glucose levels $[44,45]$ and is an expression quantitative trait locus (eQTL). By using Affymetrix Human array to analyze gene expression in 152 samples of liver tissues, Folkersen et al. [46] showed that the LIPC gene is the only gene located close to the SNP rs1532085, and its minor allele is associated with lower LIPC expression. The SNP rs1532085 is also an eQTL that interacts with other lipid metabolism genes to determine HDL-C levels and is a risk allele for Alzheimer disease and polypoidal choroidal vasculopathy [47-49]. Although these SNPs have a close relationship with lipids, our data demonstrated that their association with 8-OHdG levels were independent of HDL-C or TG levels because their association was not affected after the adjustment for HDL-C and TG levels. HL has been shown to affect the ROS by modulating the activity of PPARS, a key transcription factor known to counteract ROS production [16-18] and urinary 8-OHdG is generated following the repair of ROS-mediated DNA damages [50]. Thus we suggested that the LIPC variants decreased LIPC transcription and, in turn HL activity which further increased the levels of urinary 8-OHdG, possibly due to the down regulation of HL in the transcription factor PPAR $\delta$. Intriguingly, rs1800588 was not 


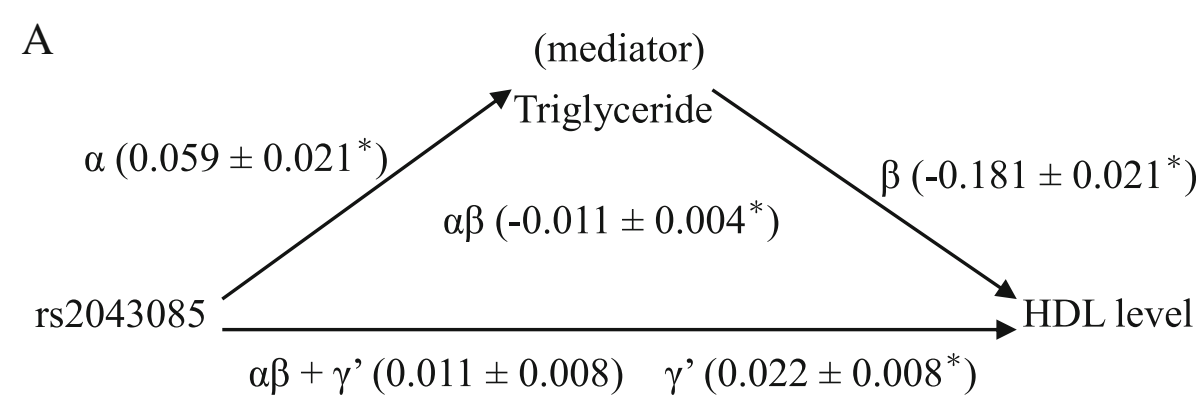

B

(mediator)

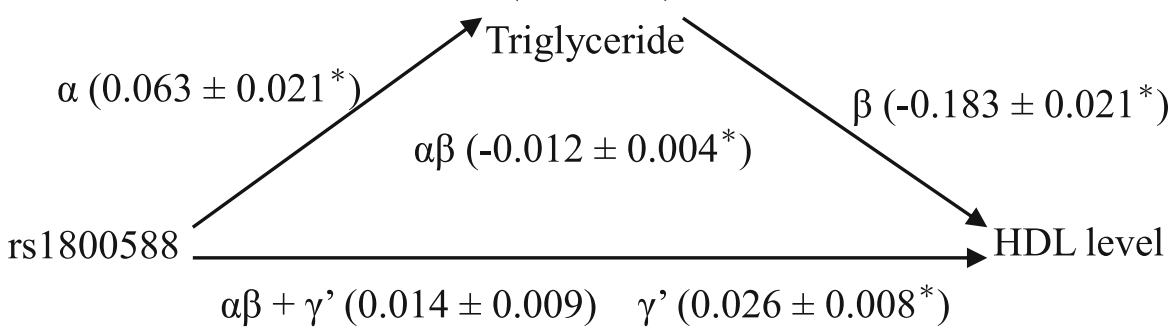

C

(mediator)

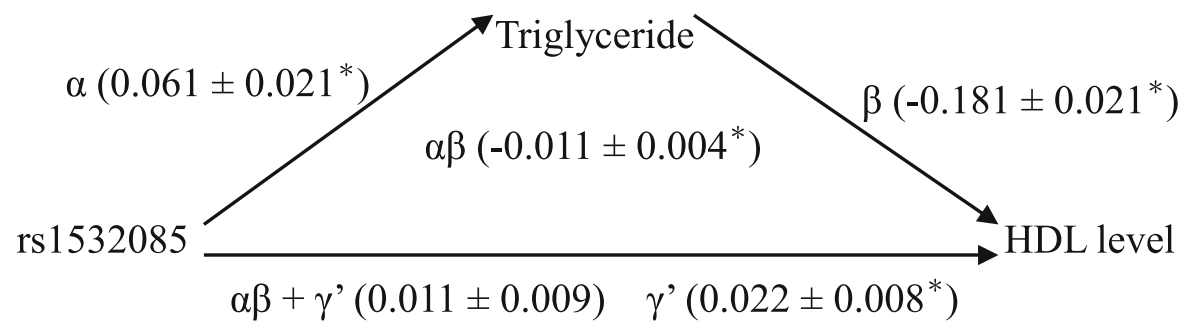

Fig. 1 A three-variable mediation model in the males with TG levels as a mediator for the associations between the LIPC genotypes and HDL-C levels. Linear regression models were used to assess the following path associations, exemplified in (a). Relationships between (a) the LIPC genotype and TG levels, ( $\beta$ ) the TG and HDL-C levels, $(a \beta+\gamma)$ the LIPC genotypes and HDL-C levels, and ( $\gamma$ ) the LIPC genotypes and HDL-C levels after adjustment for the TG levels were shown. Each estimate along the path represented the unstandardized $\beta$ coefficient from the regression model. The results indicated that the LIPC genotypes exhibited a stronger association with the HDL-C levels after adjustment for the TG levels. The direct effects ( $\gamma^{\prime}$ ) of the LIPC genotypes on the HDL-C levels (0.022) were greater than the total effects $\left(\alpha \beta+\gamma^{\prime}\right)(0.011)$, which had opposite signs to those of the mediation effects (aß) $(-0.011)$, and suggested significant mediation (suppression) by the TG levels. All models were adjusted for age, BMl, and current smoking status. ${ }^{*} P<0.01$. In addition, the other analyses (b and $\mathbf{c}$ ) exhibited similar suppression effects. Suppression triangles obtained from the male subjects

linked to 8-OHdG levels, disagreeing with a previous finding of an association with levels of MDA-LDL, an oxidative stress marker [9]. This may be attributable to the limited size of our sample population, difference in ethnicity, heterogeneity of genome complexity, or a differential requirement of HL activity for lipid and nucleic acid oxidation. Additional studies are warranted to address the origin of this discrepancy and to clarify the causal link between HL activity and 8-OHdG production. In a PheWAS study conducted by Hall et al., significant associations were observed between rs1800588 and levels of folate and vitamin E, both of which were used as antioxidants to prevent atherosclerosis [12]. In addition, two LIPC SNPs were found to be associated with advanced age-related macular degeneration (AMD) [51-53], whose progression was characterized by a hallmark of age-related oxidative changes [54]. These data suggest that the LIPC locus is a key regulator of systemic oxidative stress.

We have previously demonstrated that sex and obesity interacted with two LIPC promoter polymorphisms to determine HDL-C levels in a Taiwanese population [8]. 
In this study, we further revealed that the association of the LIPC SNPs with HDL-C and 8-OHdG levels was predominantly observed in the men and those with high adiposity status. By contrast, their associations with serum TG levels were predominantly observed in the men and nonobese participants. Our findings are in line with those of previous studies, in which associations of multiple LIPC SNPs with HDL-C levels and risks of myocardial infarction and coronary artery disease were influenced by dietary intake, physical activity, sex, and obesity $[10,14,47,55-60]$. These results suggest that the association of LIPC SNPs with various phenotypes should be individually assessed according to sex and obesity status.

In this study, we demonstrated that serum TG levels suppressed the relationship between LIPC SNPs and HDL-C levels. Several types of third variables calculated through statistical analyses, including mediation variables, confounding variables, suppression variables, and moderators [42], can be used to address the relationship between an independent and a dependent variable. Suppression variables increase the predictive validity of another variable by its inclusion into a regression equation. In general, the omission of a suppressor would underestimate the effect of an independent variable on a dependent variable, thereby reducing the magnitude of the relationship between the two variables. We have previously reported that this suppression effect may be biologically crucial, and it may partially explain the missing heritability in healthy individuals $[38,61,62]$. In brief, we previously demonstrated a suppression effect of sE-selectin levels on the association of $A B O$ blood group genotypes with the TG-to-HDL-C ratio [38]. Furthermore, we demonstrated that adiponectin levels suppressed the association of $\mathrm{CDH} 13$ genotypes with metabolic phenotypes, and the respective acute phase reaction protein levels reciprocally suppressed the association of $C R P / S A A$ genotypes with SAA/CRP levels $[61,62]$. The demonstration of the suppression effects of serum TG levels on the association of the LIPC SNPs with HDL-C levels in the men support the crucial role of the suppression effect in genetic association studies and further suggests that suppression is dependent on sex.

Pleiotropy observed in genetic association studies can provide insight into the shared biology underlying a spectrum of phenotypes. The existence of a real form of pleiotropy may be considered when associations with multiple phenotypes are observed on the level of an SNP, a gene, or a locus [63]. A recent study of the National Human Genome Research Institute's catalogue of published genome-wide association studies reported that $4.6 \%$ of the SNPs and $16.9 \%$ of the genes have cross-phenotype effects [64]. However, these are believed to be underestimated because they rely on highly conservative criteria. Pleiotropy for LIPC SNPs has been widely reported. For instance, by resequencing the coding and the $5^{\prime}$ and $3^{\prime}$ untranslated regions of 78 candidate genes, Service et al. reported the association of LIPC SNPs with TC, HDL-C, and TG levels [65], which are the most commonly found associations for LIPC SNPs. In addition, different LIPC SNPs have been associated with levels of circulating MDA-LDL [9]; phospholipids and sphingolipids [66]; folate and vitamin E [12]; metabolic syndrome [44]; visceral adiposity indicators (VAI) and triglyceride and fasting plasma glucose (TyG) index-related parameters [37]; and diseases such as advanced AMD [52, 53, 67], coronary artery disease [10], and myocardial infarction [55]. Our finding of an association of the LIPC SNPs with urinary 8-OHdG levels contributes to the growing list of these pleiotropic associations and indicates that systemic oxidative stress is a likely underlying cause of HL-associated diseases. Therefore, future in-depth research on the outcomes of this oxidative stress is essential to prevent cardiovascular disease progression in high-risk populations.

\section{Limitations}

The main limitation of this study is its moderate sample size with a relatively low number of subjects genotyped. Nevertheless, replication in a second cohort can improve the strength of the study, and the results regarding the association with HDL-C levels and interaction with sex and obesity were similar to those reported in our previous study. Another limitation is that we analyzed only three LIPC SNPs, which were incomplete and did not represent all the genetic variations of LIPC. In addition, multiple testing imposed another limitation on our study. Nevertheless, the statistical significance of a portion of our results with adjustment for the three studied SNPs became marginal when the Bonferroni correction was applied stringently to multiple tests.

\section{Conclusion}

Our study results revealed the complexity of the association of variation of the LIPC locus with lipid profiles and oxidative stress, and the crucial effects of interaction and mediation on such associations. The association of the LIPC SNPs with urinary 8-OHdG levels may contribute to our understanding of the link between various LIPC SNPs and oxidative stress-related diseases such as advanced AMD. Thus, our findings can possibly provide a way to identify high-risk populations of cardiovascular diseases for preventive medicine. Subsequent effects on treatment procedures and prognoses are expected to be derived from this study. 


\section{Additional file}

Additional file 1: Table S1. Basic characteristics and genotyping assays for the LIPC gene variants. Table S2. Associations between the LIPC rs2043085 genotypes and metabolic traits and inflammatory marker levels. Table S3. Associations between the LIPC rs1532085 genotypes and metabolic traits and inflammatory marker levels. Table S4. Associations between the LIPC rs1800588 genotypes and metabolic traits and inflammatory marker levels. Additional file References. (DOC 118 kb)

\section{Abbreviations}

8-OHdG: 8-hydroxy deoxyguanosine.; BMI: Body mass index; CRP: C-reactive protein; HDL-C: High-density lipoprotein cholesterol; HOMA-IR: Homeostasis model assessment of insulin resistance; LDL-C: Low-density lipoprotein cholesterol; MCP-1: Monocyte chemotactic protein-1; MMP1: Matrix metalloproteinase 1; MMP2: Matrix metalloproteinase 2; MMP9: Matrix metalloproteinase 9; SAA: Serum amyloid A; sE-selectin: soluble E-selectin; sICAM1: soluble intercellular adhesive molecule 1; SNPs: Single nucleotide polymorphisms; sP-selectin: soluble P-selectin; sTNFRIl: soluble tumor necrosis factor-alpha receptor 2; sVCAM1: soluble vascular cell adhesive molecule 1; TG: Triglycerides

\section{Acknowledgements}

We greatly appreciate technical support from the Core Laboratory of the Taipei Tzu Chi Hospital, Buddhist Tzu Chi Medical Foundation, and expert statistical analysis assistance from Dr. I-Shiang Tzeng.

\section{Funding}

This study was supported by grants from the Taipei Tzu Chi Hospital, Buddhist Tzu Chi Medical Foundation (TCRD-TPE-103-RT-2, TCRD-I103-01-01, TCRD-TPE-NCS-102-01, TCRD-TPE-MOST-103-01, TCRD-TPE-MOST-104-09), grants from the Tzu Chi University, Hualien, Taiwan (TCIRP102001-02Y1, TCMMP104-06-03), grants from the National Science Council (NSC 101-2314B-303 -023 -MY3, MOST 104-2314-B-303-013-MY3) to Y. L. Ko, and grants from the Taipei Tzu Chi Hospital, Buddhist Tzu Chi Medical Foundation (TCRD-TPE-103-22, TCRD-TPE-104-18, TCRD-TPE-106-46, TCRD-TPE-106-46(2) 2)) to M. S. Teng.

\section{Availability of data and materials}

The data are within the manuscript and Additional file 1.

\section{Authors' contributions}

MST and SW participated in genotyping, performed statistical analysis and drafted the manuscript. MST prepared the DNA samples and participated in genotyping. HHC participated in sample collection and prepared the DNA samples. LAH and IST performed and corrected statistical analysis. YLK and CWS supervised the study and revised the manuscript. All authors read and approved the final version of the manuscript

\section{Ethics approval and consent to participate}

This study was approved by the Institutional Review Board of Taipei Tzu Chi Hospital, Buddhist Tzu Chi Medical Foundation. After obtaining informed consent, participants from the general population were consecutively recruited during routine cardiovascular health examinations.

\section{Consent for publication}

Not applicable.

\section{Competing interests}

The authors declare that they have no competing interests.

\section{Publisher's Note}

Springer Nature remains neutral with regard to jurisdictional claims in published maps and institutional affiliations.

\section{Author details}

'Department of Research, Taipei Tzu Chi Hospital, Buddhist Tzu Chi Medical Foundation, New Taipei city, Taiwan. ${ }^{2}$ Department of Life Science, Chinese Culture University, Taipei, Taiwan. ${ }^{3}$ The First Cardiovascular Division,
Department of Internal Medicine, Chang Gung Memorial Hospital and Chang Gung University College of Medicine, Taoyuan, Taiwan. ${ }^{4}$ The Division of Cardiology, Department of Internal Medicine and Cardiovascular Center, Taipei Tzu Chi Hospital, Buddhist Tzu Chi Medical Foundation, New Taipei city, Taiwan. ${ }^{5}$ School of Medicine, Tzu Chi University, Hualien, Taiwan.

Received: 21 November 2018 Accepted: 24 April 2019

Published online: 10 May 2019

\section{References}

1. Kobayashi J, Miyashita K, Nakajima K, Mabuchi H. Hepatic lipase: a comprehensive view of its role on plasma lipid and lipoprotein metabolism. J Atheroscler Thromb. 2015;22:1001-11.

2. Annema W, Tietge UJ. Role of hepatic lipase and endothelial lipase in highdensity lipoprotein-mediated reverse cholesterol transport. Curr Atheroscler Rep. 2011;13:257-65.

3. Cohen JC, Vega GL, Grundy SM. Hepatic lipase: new insights from genetic and metabolic studies. Curr Opin Lipidol. 1999:10:259-67.

4. Thuren T. Hepatic lipase and HDL metabolism. Curr Opin Lipidol. 2000;11: 277-83.

5. Aulchenko YS, Ripatti S, Lindqvist I, Boomsma D, Heid IM, Pramstaller PP, et l. Loci influencing lipid levels and coronary heart disease risk in 16 European population cohorts. Nat Genet. 2009;41:47-55.

6. Chasman DI, Pare G, Mora S, Hopewell JC, Peloso G, Clarke R, et al. Fortythree loci associated with plasma lipoprotein size, concentration, and cholesterol content in genome-wide analysis. PLoS Genet. 2009;5:e1000730.

7. Lu X, Huang J, Mo Z, He J, Wang L, Yang X, et al. Genetic susceptibility to lipid levels and lipid change over time and risk of incident hyperlipidemia in Chinese populations. Circ Cardiovasc Genet. 2016;9:37-44.

8. Ko YL, Hsu LA, Hsu KH, Ko YH, Lee YS. The interactive effects of hepatic lipase gene promoter polymorphisms with sex and obesity on high-densitylipoprotein cholesterol levels in Taiwanese-Chinese. Atherosclerosis. 2004; 172:135-42

9. Yamazaki K, Bujo H, Taira K, Itou N, Shibasaki M, Takahashi K, et al. Increased circulating malondialdehyde-modified LDL in the patients with familial combined hyperlipidemia and its relation with the hepatic lipase activity. Atherosclerosis. 2004;172:181-7.

10. Zhang C, Lopez-Ridaura R, Rimm EB, Li T, Hunter DJ, Hu FB. Genetic variation in the hepatic lipase gene and the risk of coronary heart disease among US diabetic men: potential interaction with obesity. Diabetologia. 2006:49:1552-9.

11. Shohet RV, Vega GL, Bersot TP, Mahley RW, Grundy SM, Guerra R, et al Sources of variability in genetic association studies: insights from the analysis of hepatic lipase (LIPC). Hum Mutat. 2002;19:536-42.

12. Hall MA, Verma A, Brown-Gentry KD, Goodloe R, Boston J, Wilson S, et al. Detection of pleiotropy through a phenome-wide association study (PheWAS) of epidemiologic data as part of the environmental architecture for genes linked to environment (EAGLE) study. PLoS Genet. 2014;10: e1004678.

13. Isaacs A, Sayed-Tabatabaei FA, Njajou OT, Witteman JC, van Duijn CM. The -514 C $->$ T hepatic lipase promoter region polymorphism and plasma lipids: a meta-analysis. J Clin Endocrinol Metab. 2004:89:3858-63.

14. Ma L, Brautbar A, Boerwinkle E, Sing CF, Clark AG, Keinan A. Knowledgedriven analysis identifies a gene-gene interaction affecting high-density lipoprotein cholesterol levels in multi-ethnic populations. PLoS Genet. 2012; 8:e1002714.

15. Jialal I, Vega GL, Grundy SM. Physiologic levels of ascorbate inhibit the oxidative modification of low density lipoprotein. Atherosclerosis. 1990;82: 185-91.

16. Brown JD, Oligino E, Rader DJ, Saghatelian A, Plutzky J. VLDL hydrolysis by hepatic lipase regulates PPARdelta transcriptional responses. PLoS One. 2011;6:e21209

17. Tesse A, Andriantsitohaina R, Ragot T. PPARdelta activity in cardiovascular diseases: a potential pharmacological target. PPAR Res. 2009:745821.

18. Lee H, Ham SA, Kim MY, Kim JH, Paek KS, Kang ES, et al. Activation of PPARdelta counteracts angiotensin II-induced ROS generation by inhibiting rac1 translocation in vascular smooth muscle cells. Free Radic Res, 2012:46: 912-9.

19. Aviram M. Modified forms of low density lipoprotein and atherosclerosis. Atherosclerosis. 1993;98:1-9. 
20. Zambon A, Deeb SS, Pauletto P, Crepaldi G, Brunzell JD. Hepatic lipase: a marker for cardiovascular disease risk and response to therapy. Curr Opin Lipidol. 2003;14:179-89.

21. Waterworth DM, Ricketts SL, Song K, Chen L, Zhao JH, Ripatti S, Aulchenko YS, et al. Genetic variants influencing circulating lipid levels and risk of coronary artery disease. Arterioscler Thromb Vasc Biol. 2010;30:2264-76.

22. Cooke MS, Evans MD, Herbert KE, Lunec J. Urinary 8-oxo-2'deoxyguanosine-- source, significance and supplements. Free Radic Res. 2000;32:381-97.

23. Calderon-Garciduenas $L$, Wen-Wang $L$, Zhang YJ, Rodriguez-Alcaraz A, Osnaya N, Villarreal-Calderon A, et al. 8-hydroxy-2'-deoxyguanosine, a major mutagenic oxidative DNA lesion, and DNA strand breaks in nasal respiratory epithelium of children exposed to urban pollution. Environ Health Perspect. 1999;107:469-74.

24. Di Minno A, Turnu L, Porro B, Squellerio I, Cavalca V, Tremoli E, et al. 8Hydroxy-2-Deoxyguanosine levels and cardiovascular disease: a systematic review and meta-analysis of the literature. Antioxid Redox Signal. 2016;24: 548-55.

25. Wu LL, Chiou CC, Chang PY, Wu JT. Urinary 8-OHdG: a marker of oxidative stress to DNA and a risk factor for cancer, atherosclerosis and diabetics. Clin Chim Acta. 2004;339:1-9.

26. Nakamura T, Fujiwara N, Sugaya T, Ueda Y, Koide H. Effect of red wine on urinary protein, 8-hydroxydeoxyguanosine, and liver-type fatty acid-binding protein excretion in patients with diabetic nephropathy. Metabolism. 2009; 58:1185-90.

27. Loft S, Vistisen K, Ewertz M, Tjonneland A, Overvad K, Poulsen HE. Oxidative DNA damage estimated by 8-hydroxydeoxyguanosine excretion in humans: influence of smoking, gender and body mass index. Carcinogenesis. 1992; 13:2241-7.

28. Dandona P, Thusu K, Cook S, Snyder B, Makowski J, Armstrong D, et al. Oxidative damage to DNA in diabetes mellitus. Lancet. 1996;347:444-5.

29. Griendling KK, FitzGerald GA. Oxidative stress and cardiovascular injury: part I: basic mechanisms and in vivo monitoring of ROS. Circulation. 2003;108: 1912-6.

30. Wiseman $\mathrm{H}$, Halliwell B. Damage to DNA by reactive oxygen and nitrogen species: role in inflammatory disease and progression to cancer. Biochem J. 1996;313 ( Pt 1:17-29.

31. Sakano N, Takahashi N, Wang DH, Sauriasari R, Takemoto K, Kanbara S, et al. Plasma 3-nitrotyrosine, urinary 8-isoprostane and 8-OHdG among healthy Japanese people. Free Radic Res. 2009;43:183-92.

32. Hasham SN, Pillarisetti S. Vascular lipases, inflammation and atherosclerosis Clin Chim Acta. 2006;372:179-83.

33. Chiu HK, Qian K, Ogimoto K, Morton GJ, Wisse BE, Agrawal N, et al. Mice lacking hepatic lipase are lean and protected against diet-induced obesity and hepatic steatosis. Endocrinology. 2010;151:993-1001.

34. Andrés-Blasco I, Herrero-Cervera A, Vinué Á, Martínez-Hervás S, Piqueras $L$, Sanz MJ, et al. Hepatic lipase deficiency produces glucose intolerance, inflammation and hepatic steatosis. J Endocrinol. 2015;227: 179-91.

35. Ridker PM, Buring JE, Cook NR, Rifai N. C-reactive protein, the metabolic syndrome, and risk of incident cardiovascular events: an 8-year follow-up of 14,719 initially healthy American women. Circulation. 2003;107:391-7.

36. Hsu LA, Ko YL, Wu S, Teng MS, Chou HH, Chang CJ, et al. Association of soluble intercellular adhesion molecule-1 with insulin resistance and metabolic syndrome in Taiwanese. Metabolism. 2009;58:983-8.

37. Teng MS, Wu S, Er LK, Hsu LA, Chou HH, Ko YL. LIPC variants as genetic determinants of adiposity status, visceral adiposity indicators, and triglyceride-glucose (TyG) index-related parameters mediated by serum triglyceride levels. Diabetol Metab Syndr. 2018;10:79. https://doi.org/10.1186/ s13098-018-0383-9. eCollection 2018.

38. Teng MS, Hsu LA, Wu S, Chou HH, Chang CJ, Sun YZ, et al. Mediation analysis reveals a sex-dependent association between $A B O$ gene variants and TG/HDL-C ratio that is suppressed by sE-selectin level. Atherosclerosis. 2013;228:406-12.

39. Baron RM, Kenny DA. The moderator-mediator variable distinction in social psychological research: conceptual, strategic, and statistical considerations. J Pers Soc Psychol. 1986;51:1173-82.

40. Mackinnon DP. Analysis of mediating variables in prevention and intervention research. NIDA Res Monogr. 1994;139:127-53.

41. Sobel ME. Direct and indirect effects in linear structural equation models. Sociol Methods Res. 1987;16:155-76.
42. Mackinnon DP, Krull $J$, Lockwood CM. Equivalence of the mediation, confounding and suppression effect. Prev Sci. 2000;1:173-81.

43. Sobel ME. Asymptotic confidence intervals for indirect effects in structural equation models. Sociol Methodol. 1982;13:290-312.

44. Kraja AT, Vaidya D, Pankow JS, Goodarzi MO, Assimes TL, Kullo IJ, et al. A bivariate genome-wide approach to metabolic syndrome: STAMPEED consortium. Diabetes. 2011;60:1329-39.

45. Zhou L, He M, Mo Z, Wu C, Yang H, Yu D, et al. A genome wide association study identifies common variants associated with lipid levels in the Chinese population. PLoS One. 2013:8:e82420.

46. Folkersen L, van't Hooft F, Chernogubova E, Agardh HE, Hansson GK, Hedin $U$, et al. Association of genetic risk variants with expression of proximal genes identifies novel susceptibility genes for cardiovascular disease. Circ Cardiovasc Genet. 2010;3(4):365-73.

47. Ma L, Ballantyne C, Brautbar A, Keinan A. Analysis of multiple association studies provides evidence of an expre ssion QTL hub in gene-gene interaction network affecting HDL cholesterol levels. PLoS One. 2014;9: e92469.

48. Meng Q, Huang L, Sun Y, Bai Y, Wang B, Yu W, et al. Effect of highdensity lipoprotein metabolic pathway gene variations and risk factors on neovascular age-related macular degeneration and polypoidal choroidal vasculopathy in China. PLoS One. 2015;10:e0143924.

49. Simmons CR, Zou F, Younkin SG, Estus S. Evaluation of the global association between cholesterol-associated polymorphisms and Alzheimer's disease suggests a role for rs3846662 and HMGCR splicing in disease risk. Mol Neurodegener. 2011;6:62.

50. Evans MD, Dizdaroglu M, Cooke MS. Oxidative DNA damage and disease: induction, repair and significance. Mutat Res. 2004;567(1):1-61 Review.

51. Chen W, Stambolian D, Edwards AO, Branham KE, Othman M, Jakobsdottir J, et al. Genetic variants near TIMP3 and high-density lipoprotein-associated loci influence susceptibility to age-related macular degeneration. Proc Natl Acad Sci U S A. 2010;107:7401-6.

52. Neale BM, Fagerness J, Reynolds R, Sobrin L, Parker M, Raychaudhuri S, et al. Genome-wide association study of advanced age-related macular degeneration identifies a role of the hepatic lipase gene (LIPC). Proc Natl Acad Sci U S A. 2010;107:7395-400.

53. Seddon JM, Reynolds R, Rosner B. Associations of smoking, body mass index, dietary lutein, and the LIPC gene variant rs10468017 with advanced age-related macular degeneration. Mol Vis. 2010;16:2412-24.

54. Jarrett SG, Boulton ME. Consequences of oxidative stress in age-related macular degeneration. Mol Asp Med. 2012;33:399-417.

55. Ahmad T, Chasman DI, Buring JE, Lee IM, Ridker PM, Everett BM. Physical activity modifies the effect of LPL, LIPC, and CETP polymorphisms on HDL-C levels and the risk of myocardial infarction in women of European ancestry. Circ Cardiovasc Genet. 2011;4:74-80.

56. Grarup N, Andreasen CH, Andersen MK, Albrechtsen A, Sandbaek A, Lauritzen $T$, et al. The $-250 \mathrm{G}>$ a promoter variant in hepatic lipase associates with elevated fasting serum high-density lipoprotein cholesterol modulated by interaction with physical activity in a study of 16,156 Danish subjects. J Clin Endocrinol Metab. 2008;93:2294-9.

57. Lopez-Rios L, Novoa FJ, Chirino R, Varillas F, Boronat-Cortes M, Wagner AM. Interaction between cholesteryl ester transfer protein and hepatic lipase encoding genes and the risk of type 2 diabetes: results from the Telde study. PLoS One. 2011;6:e27208.

58. St-Pierre J, Miller-Felix I, Paradis ME, Bergeron J, Lamarche B, Despres JP, et al. Visceral obesity attenuates the effect of the hepatic lipase -514C>T polymorphism on plasma HDL-cholesterol levels in French-Canadian men. Mol Genet Metab. 2003;78:31-6.

59. Yin RX, Wu DF, Miao L, Aung LH, Cao XL, Yan TT, et al. Several genetic polymorphisms interact with overweight/obesity to influence serum lipid levels. Cardiovasc Diabetol. 2012;11:123.

60. Zhang C, Lopez-Ridaura R, Rimm EB, Rifai N, Hunter DJ, Hu FB. Interactions between the -514C->T polymorphism of the hepatic lipase gene and lifestyle factors in relation to HDL concentrations among US diabetic men. Am J Clin Nutr. 2005:81:1429-35.

61. Ko YL, Hsu LA, Wu S, Teng MS, Chou HH. CRP and SAA1 haplotypes are associated with both C-reactive protein and serum amyloid a levels: role of suppression effects. Mediat Inflamm. 2016;2016:12.

62. Teng MS, Hsu LA, Wu S, Sun YC, Juan SH, Ko YL. Association of CDH13 genotypes/haplotypes with circulating adiponectin levels, metabolic 
syndrome, and related metabolic phenotypes: the role of the suppression effect. PLoS One. 2015;10:e0122664.

63. Solovieff N, Cotsapas C, Lee PH, Purcell SM, Smoller JW. Pleiotropy in complex traits: challenges and strategies. Nat Rev Genet. 2013;14:483-95.

64. Sivakumaran S, Agakov F, Theodoratou E, Prendergast JG, Zgaga L, Manolio $T$, et al. Abundant pleiotropy in human complex diseases and traits. Am J Hum Genet. 2011;89:607-18.

65. Service SK, Teslovich TM, Fuchsberger C, Ramensky V, Yajnik P, Koboldt DC, et al. Re-sequencing expands our understanding of the phenotypic impact of variants at GWAS loci. PLoS Genet. 2014;10:e1004147.

66. Demirkan A, van Duijn CM, Ugocsai P, Isaacs A, Pramstaller PP, Liebisch G, et al. Genome-wide association study identifies novel loci associated with circulating phospho- and sphingolipid concentrations. PLoS Genet. 2012;8: e1002490.

67. Cipriani V, Leung HT, Plagnol V, Bunce C, Khan JC, Shahid H, et al. Genomewide association study of age-related macular degeneration identifies associated variants in the TNXB-FKBPL-NOTCH4 region of chromosome 6p21.3. Hum Mol Genet. 2012;21:4138-50.

Ready to submit your research? Choose BMC and benefit from:

- fast, convenient online submission

- thorough peer review by experienced researchers in your field

- rapid publication on acceptance

- support for research data, including large and complex data types

- gold Open Access which fosters wider collaboration and increased citations

- maximum visibility for your research: over $100 \mathrm{M}$ website views per year

At $\mathrm{BMC}$, research is always in progress.

Learn more biomedcentral.com/submissions 